\title{
The Antiviral Activity of Andrographolide, the Active Metabolite from Andrographis paniculata (Burm. f.) Wall. ex Nees. against SARS-CoV-2 by Using Bio- and Chemoinformatic Tools
}

\section{Thanet PITAKBUT}

Technical Biochemistry Laboratory, Bio - Center, Faculty of Chemical and Biochemical Engineering, Technical University Dortmund University, Dortmund, Germany

(Corresponding author's e-mail: thanet.pitakbut@tu-dortmund.de)

Received: 3 March 2020, Revised: 4 July 2020, Accepted: 9 July 2020

\begin{abstract}
Due to the severe acute respiratory syndrome coronavirus 2 or SARS-CoV-2 outbreak, the virus has been wildly spread throughout the world and the number of infected patients has rapidly increased. More importantly, neither the official drug treatment nor the vaccine has been officially offered. These have considerably increased the public concerns internationally and nationally. Recently, there has been one question raised in the Thai society; "Could a common Thai herbal medicine namely Andrographis paniculata be used against SARS-CoV-2 infection?". It is well-known that the plant has antiviral properties against wild ranges of viruses and the active metabolite is andrographolide. To date, there have only been a few studies investigating the anti-SARS-CoV-2 activity from andrographolide. To provide a better understanding, this study was conducted by applying the advanced techniques in both computational biology and chemistry to evaluate the anti-SARS-CoV-2 potential of andrographolide. In this study, andrographolide was tested against two key enzymes of SAR-CoV-2 namely 3C main proteinase and RNA dependent RNA polymerase. The result here indicated that andrographolide could only inhibit the SARS-CoV-2 3C main proteinase as strong as lopinavir (the standard medicine), which has been recommended as the drug of choice to treat SARS-CoV-2 patient.
\end{abstract}

Keywords: Antiviral activity, SARS-CoV-2, Andrographolide, Andrographis paniculata, Computational techniques

\section{Introduction}

Since the $1^{\text {st }}$ outbreak in Wuhan, China, at the end of 2019 , the virus that caused this outbreak had been identified and named as the severe acute respiratory syndrome coronavirus 2 or SARS-CoV-2. Thus far, this virus has infected people all over the world and the number of infections is continuously increasing. This virus is not only causing the world health problem, but also the world economy due to the speedy spread. It has become a global threat. Therefore, the World Health Organization (WHO) announced SARS-CoV-2 as the new pandemic [1,2]. Despite the international research cooperation, neither the official medicine that could be used against nor the vaccine that could prevent the SARS-CoV2 infection is yet available [3]. This situation causes public concerns, globally including the Thai society. Recently, there is one question frequently asked throughout Thailand: "Could a commonly known antiviral Thai herbal medicine, Andrographis paniculata, affect SARS-CoV-2 infection?". Since there is not yet the concrete scientific evidence regarding this specific issue, the answer to this question could not be addressed properly. However, the antiviral properties of A. paniculata are well-known as well as its bioactive metabolite, andrographolide. Up to now, there are wild ranges of the pharmacological activities of andrographolide against human pathogenic viruses had been reported. For example, andrographolide 
http://wjst.wu.ac.th

could reduce the copy of the viral DNA materials from hepatitis B and herpes simplex viruses as well as suppress the viral RNA replication from influenza A, hepatitis C, and chikungunya viruses, respectively. Furthermore, andrographolide could also inhibit hepatitis $\mathrm{C}$ viral proteinase activity [4]. Recently, there are crystal structures of SARS-CoV-2 available on the protein database [5,6]. Thus, It is now possible to determine the anti-SARS-CoV-2 activity from andrographolide by using the computational method. Since several medicines that are currently used to treat many illnesses clinically was originally verified by this computational approach such as zanamivir for influenza and nelfinavir as well as saquinavir for HIV [7]. Therefore, this study was conducted by applying the same computational methodology to provide the preliminary evaluation of the potential of andrographolide against SARS-CoV-2, which could be used to address the question as mentioned earlier and to provide the essential ground information for further investigations.

It is vital to understand the life-cycle of the SARS-CoV-2 in the human cell as shown in Figure 1. The virus is likely to enter the human body through the respiratory system. After the arrival at the target site, the spike protein on the membrane of SARS-CoV-2 would interact with the angiotensin-converting enzyme II (ACE-II) receptor at the surface membrane of the target cell, which would allow the virus to enter the cell. Some of the single-stranded RNA or ssRNA would be translated to the polyproteins by the host's ribosome. These polyproteins would be hydrolyzed to provide the required protein components for the viral reproduction by the $3 \mathrm{C}$ main proteinase as could be seen as proteinase in Figure $\mathbf{1}$ and this $3 \mathrm{C}$ main proteinase would be called as proteinase from now on. While the other ssRNA would be replicated by RNA dependent RNA polymerase or RdRp. Finally, the replicated ssRNA together with the hydrolyzed protein components would be assembled and the new SARS-CoV-2 viruses would be reproduced. These steps would be performed repeatedly until their host develops the illness as the socalled coronavirus disease 2019 or COVID-19 [8]. As discussed earlier, to stop the COVID-19 is to breakdown the life-cycle of this virus. Therefore, the SARS-CoV-2 proteinase and RdRp were selected as the targets in this study.

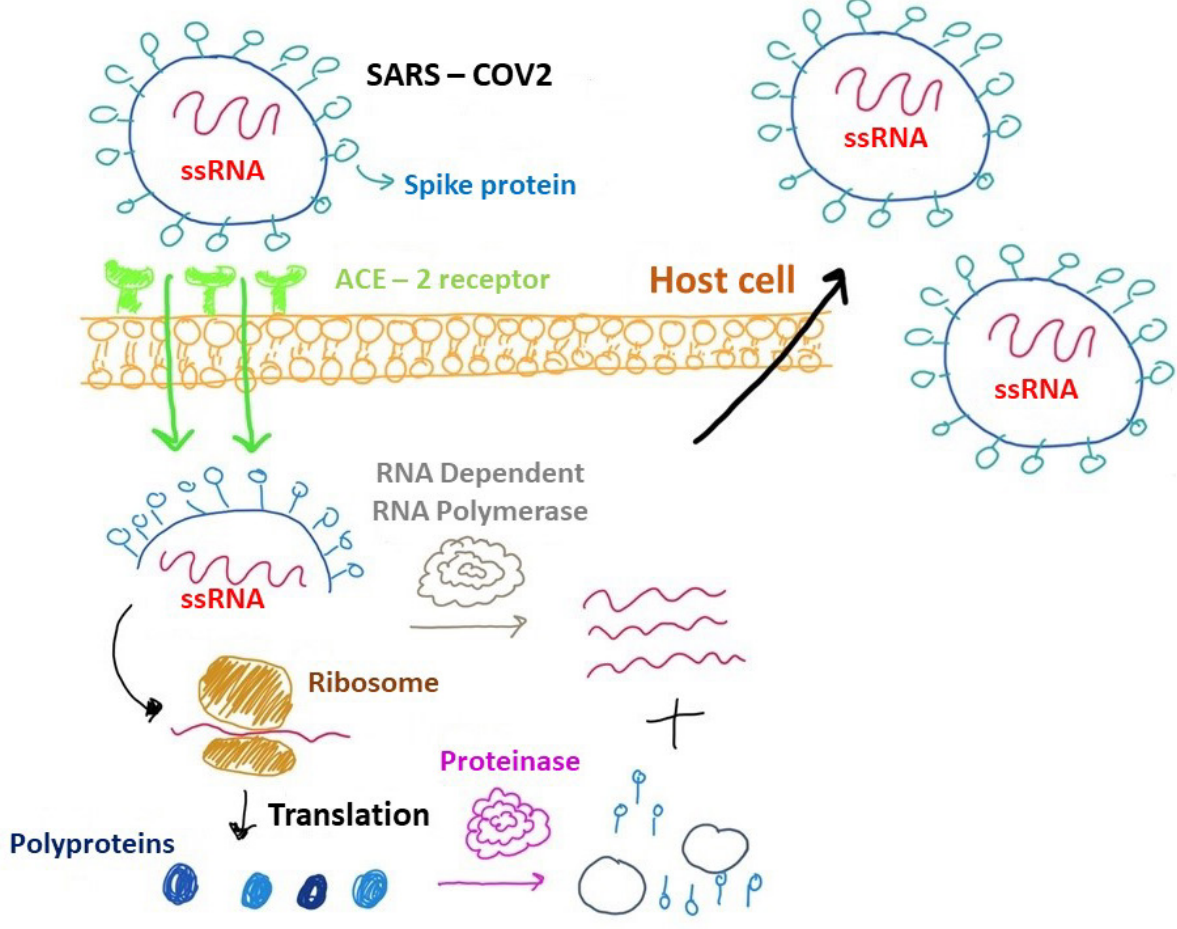

Figure 1 Simplified life-cycle of SARS-CoV-2 infection in the human cell. 
http://wjst.wu.ac.th

\section{Materials and methods}

The workflow of the research methodology that had been executed in this study was shown in Figure 2. As seen in the figure below, the workflow could be simply divided into 3 steps, which represented in 3 different boxes and colors. The $1^{\text {st }}$ step (as presented in the green box) was the preparation step for the SARS-CoV-2 proteinase and RdRp by searching for the enzymes through the protein databank, and the target enzymes were downloaded, accordingly. After that, the $2^{\text {nd }}$ step (in the yellow box) was the identification of the binding site and the method validation step, which performed by locating the binding pocket in the enzyme, designing, and validating the molecular docking protocol. The last step (in the red box) was the experimenting step. The details of each step would be described in the following section.

\section{Identification and preparation of the SARS-CoV-2 proteinase and RNA dependent RNA polymerase (RdRp)}

The SARS-CoV-2 proteinase (PDB ID: 6LU7) was downloaded from the RSCB protein databank (https://www.rcsb.org), while the SARS-CoV-2 RdRp is not yet available in the databank. Therefore, the alternative approach had been applied to overcome this challenge as mentioned in Figure 2 (in the green box). The amino acid sequences of the SARS-CoV-2 RdRp had been extracted from the open reading frame or ORF1ab polyproteins (GenBank: QHD43415.1), which was deposited in the NCBI database (https://www.ncbi.nlm.nih.gov), to use as the searching template. Notepad++ version 7.8 .5 was used to open the ORF $1 \mathrm{ab}$ polyproteins as a simple text file and to extract the amino acid sequences of the SARSCoV-2 RdRp [8]. After that, the online protein blast program from the NCBI database (https://blast.ncbi.nlm.nih.gov/Blast.cgi) was used to search for the homolog RdRp. As anticipated, the SARS-CoV-1 RdRp (PDB ID: 6NUR) had shown the highest percent identity as higher as $96 \%$, which passed the minimum criteria as shown in Figure 2. Therefore, SARS-CoV-1 RdRp was downloaded. Consequently, ClustalW package from the Seaview version 3.2 was selected to perform and visualize the alignment of the pairwise amino acid sequences [10]. Finally, the SARS-CoV-1 RdRp was used as the target instead of the RdRp from SARS-CoV-2.

\section{docking}

Navigation of the binding pocket, experimental setup and method validation for the molecular

AutodockTools version 1.5.6 was used to locate the binding pocket and set up the interaction site in the selected enzymes [11]. While USFC Chimera version 1.11.2 was chosen to visualize, delete water molecules, and extract the native ligand from the enzymes [12]. As presented in Figure 2 (the yellow box), the native ligands must be used as the navigator to identify the binding pocket in the enzymes. If there was no native ligand within the enzyme, the alternative enzyme with an available ligand had to be used instead. Therefore, the RdRp from the poliovirus (PDB ID: 2ILY) with ATP as the native ligand was selected to assist the navigation of the binding pocket in the SAR-CoV-1 RdRp due to the fact that had been discussed earlier. More details regarding this step were not presented here in this report but it would rather be provided separately in the supplementary file.

Autodock Vina Version 1.1.2 was chosen to perform the molecular docking experiment [13]. Almost all parameters from Autodock Vina were set as a default value. Only 2 parameters were adapted. One parameter was the exhaustiveness, which was adjusted up to 32 and the other parameter was the num modes, which was changed to 20 . The grid box and its size were set up specifically for each enzyme. For SARS-CoV-2 proteinase, the grid box was set as $\mathrm{X}=-13.2, \mathrm{Y}=7.2$ and $\mathrm{Z}=70.2$ with the size of $28 \times 28 \times 28$ Angstrom. On the other hand, the grid box for the SARS-CoV-1 RdRp was set as $\mathrm{X}=$ $287.3, \mathrm{Y}=43.4$ and $\mathrm{Z}=-8.5$ with the size of $18 \times 18 \times 10$ Angstrom.

Before these setup docking protocols were subjected to investigate the enzyme inhibitory activity of andrographolide, they had to be validated by redocking the extracted native ligands back into the binding pocket. Only the protocol that could provide a good correlation between the native ligand before and after the redocking experiment would be allowed to perform the investigation in this study as demonstrated in 
http://wjst.wu.ac.th

Figure 2 (the yellow color). The results of these validations would also be presented in the supplementary file.

Preparation of andrographolide and standard drugs, and performing the molecular docking experiment

All compounds that had been used in this study were downloaded from the PubChem database (https://pubchem.ncbi.nlm.nih.gov). While andrographolide and lopinavir were already in their active forms, but favipiravir and remdesivir were not yet since they were in their prodrug forms. Therefore, $\mathrm{ACD} /$ Chemsketch free version 1.0 had been used to manipulate these 2 standard drugs by adding the ribose moiety and phosphate groups into their structures [14]. After that, andrographolide, lopinavir, and the active forms of favipiravir and remdesivir were subjected to Avogadro version 1.2.0 for the geometrical optimization and the energy minimization (MMFF94 force field) [15]. AutodockTools version 1.5.6 was used once again for the final preparation before performing the molecular docking experiment as presented in Figure 2 (the red box) [11]. For the post-docking analysis, Discovery Studio Visualizer, a free version, was selected to create the molecular diagram [16].

\section{Results and discussion}

\section{Identification of the homologous RdRp of SARS-CoV-2 enzyme}

Until now, there are only a few proteins from the SARS-CoV-2 that are available in the protein databank unlike the genomic data, which has comprehensively studied and been continuously deposited in the database. Unfortunately, one of the key proteins like RdRp, which is responsible for the replication of the ssRNA, from SARS-CoV-2 is not yet available. Therefore, it was not possible to investigate the RdRp from the SARS-CoV-2 directly. To overcome this challenge, searching for the homolog enzyme that had a similar protein structure, which was available in the database was the reasonable option as described in Figure 2. To accomplish this, the amino acid sequences that encoded the RdRp from the SARS-CoV-2 at the ORF 1ab polyproteins were extracted. After that, these amino acid sequences were blasted against the protein databank. Undoubtedly, the best hit was the RdRp from the SARS-CoV-1, the closest species to SARS-CoV-2, which showed the highest percent identity as high as $96 \%$. The alignment of these two sequences and the 3D structure of the best hit, the SARS-CoV-1 RdRp, were presented in Figures 3 and 4, respectively.

Recently, Zhan and Zhou [17] have also used the SARS-CoV-1 RdRp as the template for the protein modeling to create the SARS-CoV-2 RdRp. Even the protein modeling could be done, the author was not convinced to model this protein from the SARS-CoV-1 since there was a report that strongly suggested that there was no difference in the term of the molecular interaction capability between these two enzymes when it came to the docking experiment [18]. Therefore, the author decided to use RdRp from SARS-CoV-1 as the target instead of its counterpart from SARS-CoV-2. However, there was one last problem regarding this SARS-CoV-1 RdRp. The SARS-CoV-1 RdRp did not have a native ligand in its structure. Thereby, it was not possible to navigate the binding pocket in the enzyme. This would cause a systemic problem for the following steps. Fortunately, a recent study has reported the solution to this issue. Therefore, the author followed the suggestion from the previous study by using ATP from the polio $\mathrm{RdRp}$ as the navigator [16]. More detail regarding this issue was provided in the supplementary file. 


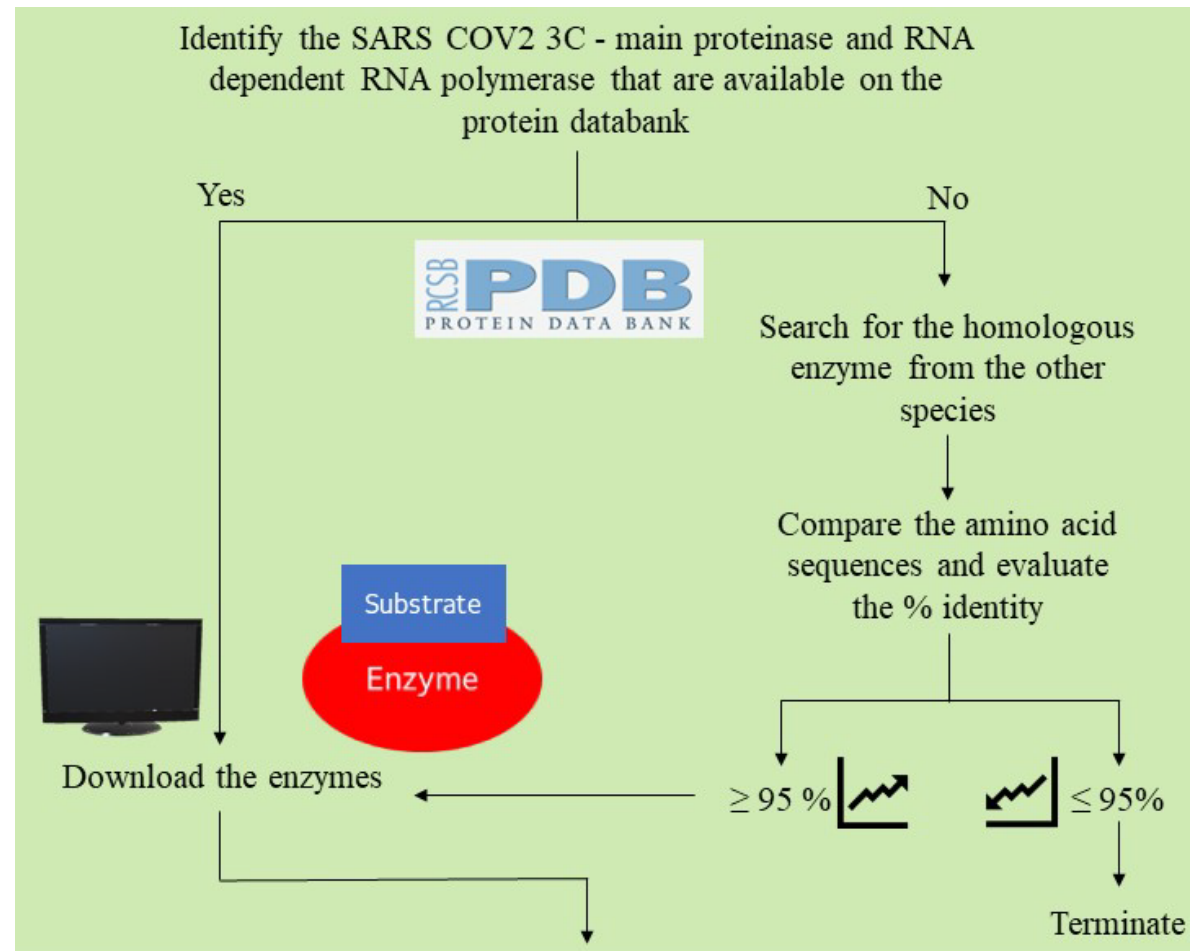

Locate the binding pocket in the enzyme by using the native ligand as the navigator
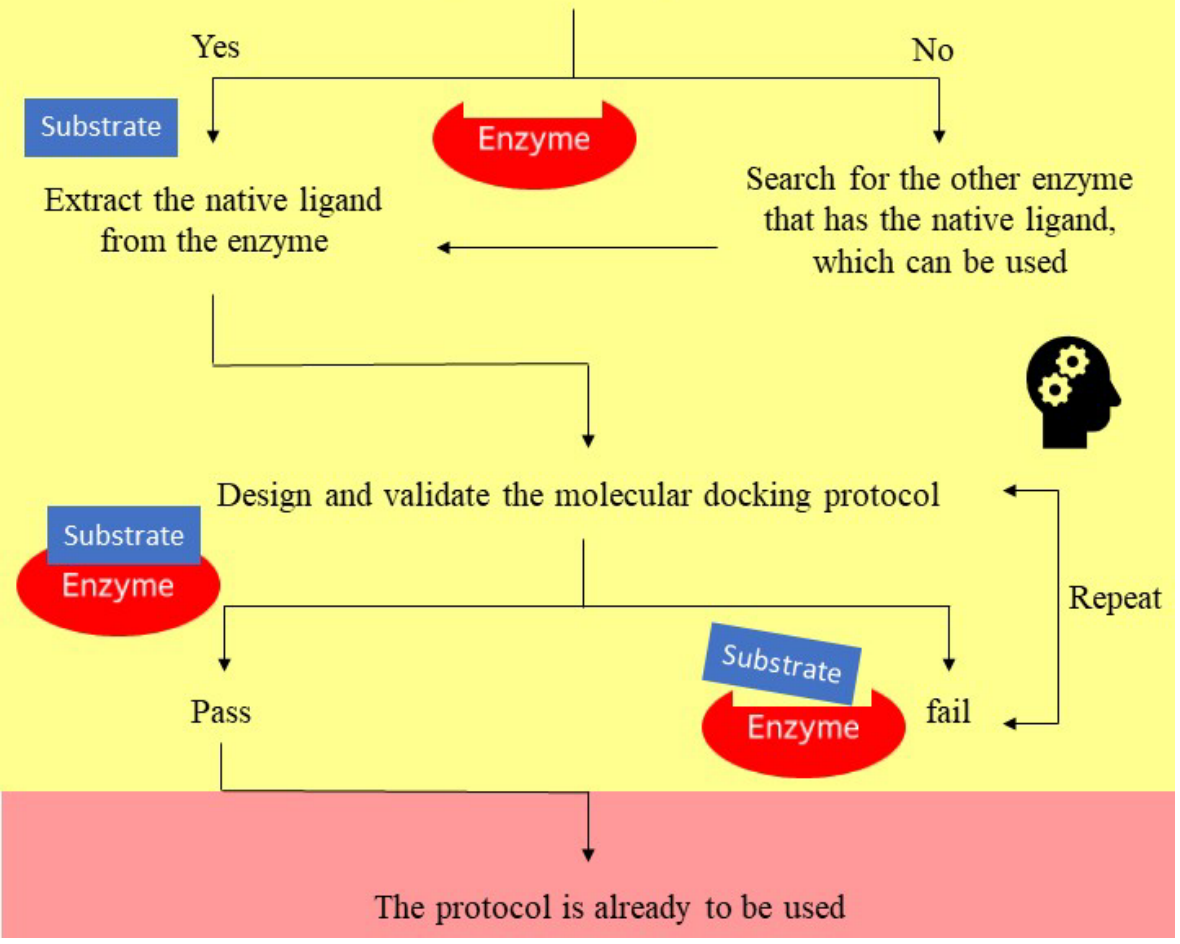

Figure 2 Overview of the research workflow that applied in this study. 


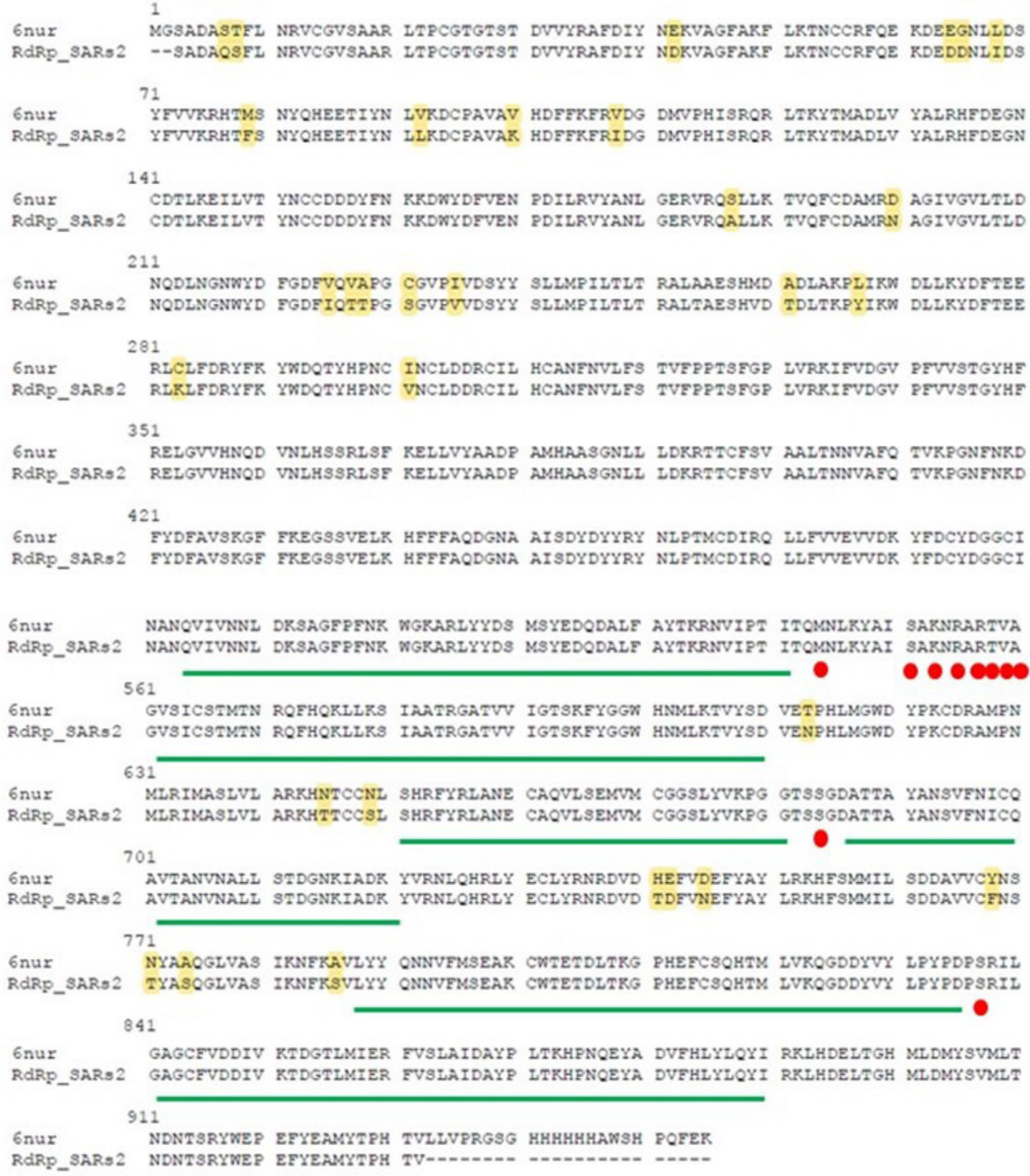

Figure 3 Pairwise alignment of the amino acid sequences between RdRp from SARS-CoV-2 (as presented in the alignment as RdRp_SARs2) and from SARS-CoV-1 (as shown as 6NUR). The red circles indicated the identical active residues between these 2 enzymes and the green lines exhibited the identical neighboring domains around the active residues. On the other hand, the yellow highlights were indicated the different amino acids between these 2 enzymes. 
http://wjst.wu.ac.th

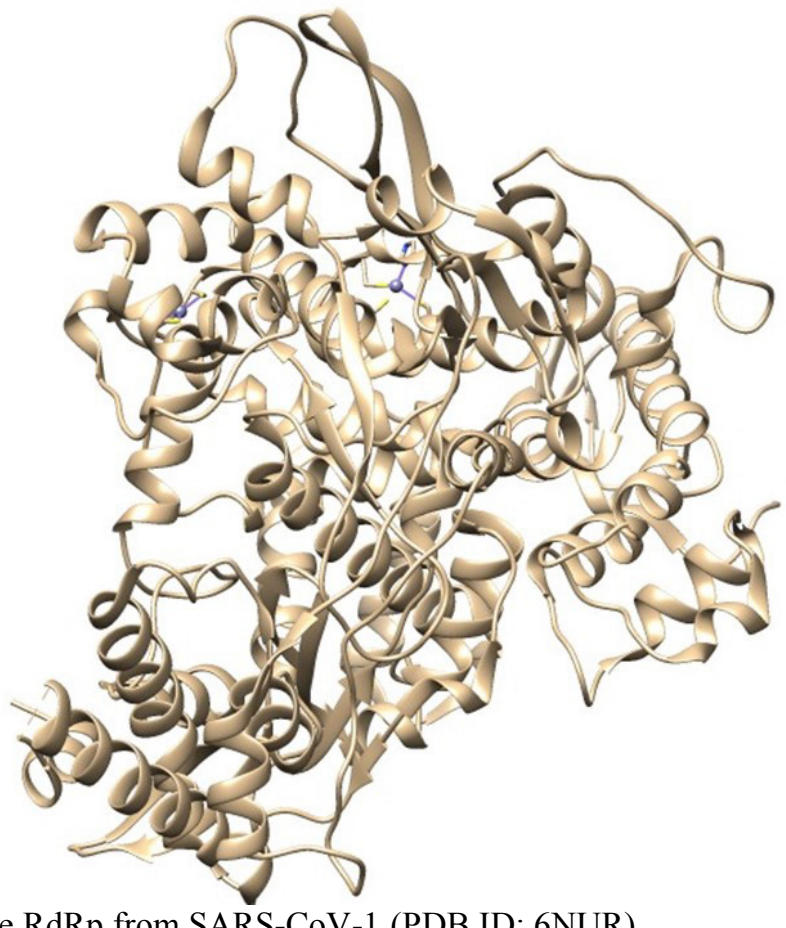

Figure 4 3D structure of the RdRp from SARS-CoV-1 (PDB ID: 6NUR).

\section{Proteinase inhibitory activity of andrographolide and lopinavir}

As described in the introduction section, SARS-CoV-2 ssRNA would be translated to the polyproteins after the virus infected the host cell. Then, these polyproteins would be hydrolyzed by the proteinase and provide the essential protein components for the virus to replicate itself. Therefore, the inhibition of this proteinase could stop the reproduction of the virus and slow down the spread of this virus to the neighboring cells. Therefore, the immune system could eliminate the virus effectively, which would eventually result in better medical improvement for the infected patient. In this study, the result revealed that andrographolide could exhibit the inhibitory activity at the same potency as lopinavir (the standard drug) as could be seen in Figures 5 and $\mathbf{6}$ below.
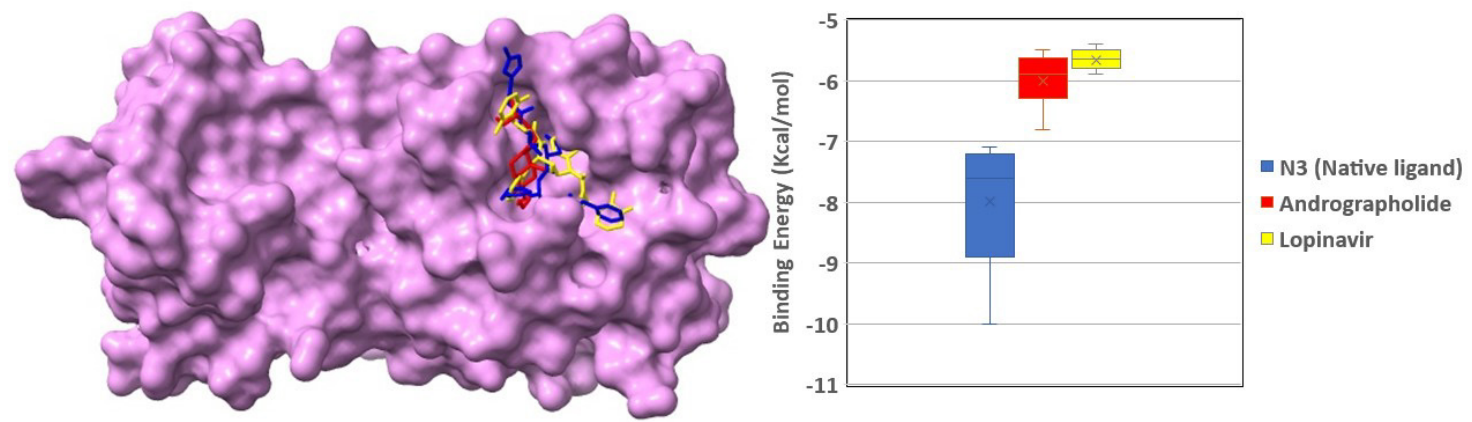

Figure 5 Molecular poses of N3 inhibitor (native ligand, blue color), andrographolide (red color) and lopinavir (standard drug, yellow color) with the SARS-CoV-2 proteinase (PDB ID: 6LUV) as presented on the left-hand side. The comparison of the average binding energy ( $\mathrm{Kcal} / \mathrm{mol})$ of N3 inhibitor, andrographolide, and lopinavir as could be seen on the right-hand side. 
http://wjst.wu.ac.th

Up to 20 conformations from the molecular docking experiments were obtained. Firstly, each of these conformations was visually evaluated to confirm that they were truly bound at the active site. The promising conformations of N3 inhibitor (blue color), andrographolide (red color) and lopinavir (yellow color) were presented in Figure 5 on the left-hand side. The result here suggested that N3 inhibitor, andrographolide, and lopinavir were binding at the same site, the active site. Secondly, the average binding energy (Kcal/mol) from all obtained conformations was calculated and presented as a box-plot, which could be seen in Figure 5 on the right-hand side. The comparison of the average binding energy between andrographolide and lopinavir showed no difference. Therefore, the data here suggested the same potency in the SARS-CoV-2 proteinase inhibitory activity between andrographolide and lopinavir.

$\mathbf{A}$

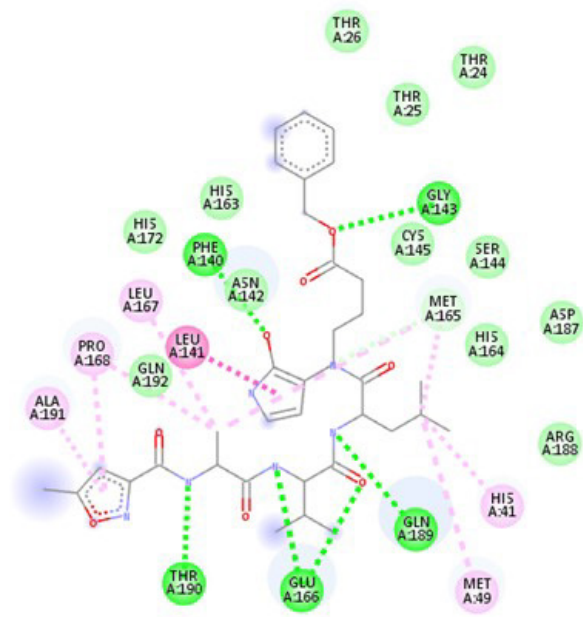

B

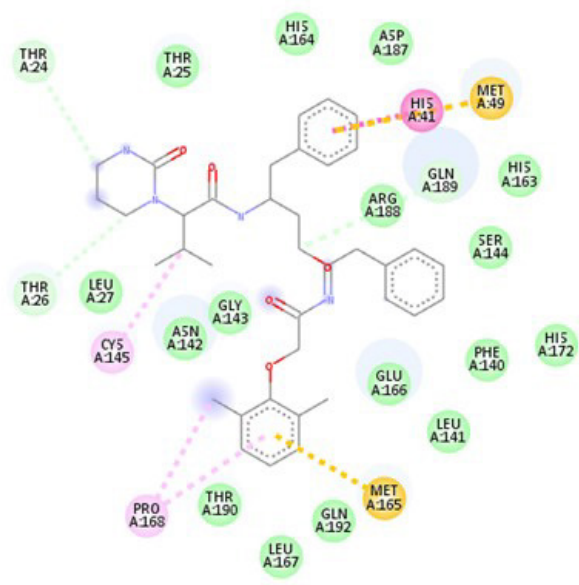

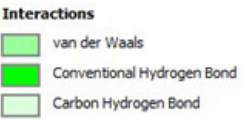
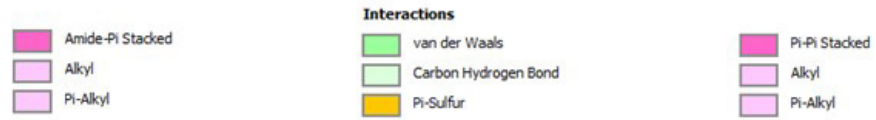

C
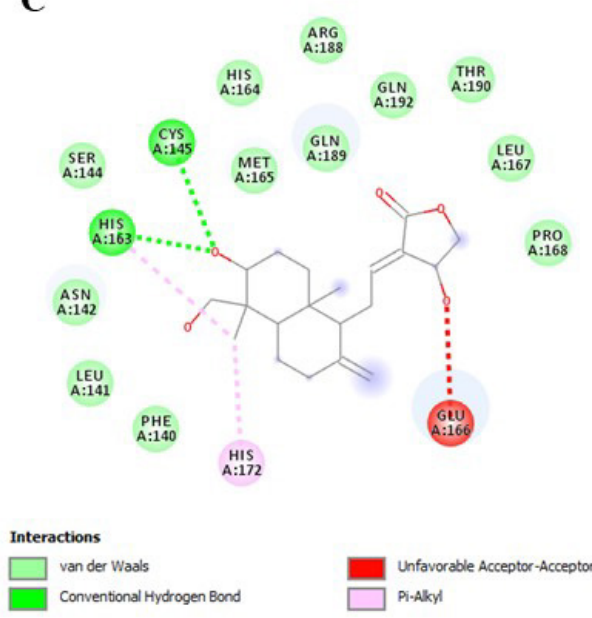

Figure 6 Interaction diagrams between SARS-CoV-2 proteinase and N3 inhibitor as a native ligand (A), lopinavir as a standard drug (B), and andrographolide (C). 
http://wjst.wu.ac.th

For a more comprehensive analysis, the interaction diagrams between SARS-CoV-2 proteinase and the compounds of interest were evaluated as presented in Figures 6A to 6C. The diagram showed the similar pattern in the molecular interactions between docked and the original pose of N3 inhibitor, the crystal structure, and SARS-CoV-2 proteinase. Only slight differences were found. The molecular interactions between N3 inhibitor and the amino acids such as Cys145, His164, and His163 from the SARS-CoV-2 proteinase were reported here from the docking experiment as hydrophobic interactions instead of hydrogen bondings as presented in the original study [19]. Nevertheless, all important amino acids involved in the binding pocket were revealed by molecular docking including Gly143, Glu166, Gln189, and Thr190, respectively. As expected, lopinavir could also bind at the same pocket as N3 inhibitor since both were competitive inhibitors to the viral proteinase. Most of the amino acids and molecular interactions that involved were quite similar to N3 inhibitor but there were some distinctive bindings, which only found in lopinavir, such as pi-sulfur and pi-alkyl interactions at Met165 and Pro168 and carbon-hydrogen bonding at Arg188, accordingly. These interactions could not be found in the N3 inhibitor. Interestingly, andrographolide could also form hydrogen bondings with Cys145, the catalytic residue, and His 163, the neighboring residue. Moreover, the other amino acids that had been found earlier in both N3 inhibitor and lopinavir such as His164, Gln166, Gln189, and Thr190 were also found here with andrographolide.

In conclusion, the obtained data in this study imply that andrographolide may have the same effect as lopinavir, the standard drug. Therefore, the author would like to purpose the potential of andrographolide against SARS-CoV-2 proteinase. As anticipated, there is a recent study reporting the derivatives of andrographolide as a promising SARS-CoV-2 proteinase inhibitor [20]. As a consequence, the result of this study is in line with the previous reports. Furthermore, Chandramohan et al, (2015) had suggested that andrographolide could also strongly inhibit the hepatitis $\mathrm{C}$ viral proteinase, which showed a similar structure to SAR-CoV-2 proteinase [21,22]. Moreover, Bafna et al, (2020) had also purposed the anti-hepatitis $\mathrm{C}$ viral proteinase drugs might be the other important source to find the cure for the SARCoV-2 infection. Therefore, it could be assumed that andrographolide may be able to against SARS-CoV2 infection by inhibiting the viral proteinase activity. Last but not less, the hepatitis $\mathrm{C}$ viral proteinase was not the only enzyme that shared similar protein structural architecture to SAR-CoV-2, but other viral proteinases from zika and dengue also had the same structure [22]. Since it was possible to observe the correlation in the anti-proteinase activities between SAR-CoV-2 and hepatitis $\mathrm{C}$ as discussed earlier, a similar phenomenon might, possibly, be applicable to the other viruses who shared the same protein architecture as well. Therefore, it would be interesting to evaluate the inhibitory activity of andrographolide against zika and dengue viral proteinases in the future.

\section{RdRp inhibitory activity of andrographolide, favipiravir and remdesivir}

As mentioned earlier, there is no crystal structure of the RdRp from SARS-CoV-2 available in the database. Therefore, the author had to search for the alternative high identical RdRp from the close species that was available in the protein database. As a result, the RdRp from SARS-CoV-1 showed the highest identity as high as $96 \%$. Thereby, SARS-CoV-1 RdRp was used as the target enyzme instead of the counterpart from SARS-CoV-2. Since the two standard drugs, favipiravir and remdesivir, were available as a prodrug in the chemical database. Therefore, these prodrugs had to be activated by adding ribose moiety and phosphate groups into their structures before subjected to the molecular docking experiment as described in the materials and methods section.

The same procedure that was applied for the proteinase was also applied here. Up to 20 conformations were collected from the experiments and each of them was confirmed visually that they were bound with RdRp. The promising conformation of ATP as the native molecule (blue color), andrographolide (red color) and the two standard drugs both favipiravir (black color) and remdesivir (yellow color) were shown in Figure 7 (left-hand side). The molecular alignment suggested that they all bound with RdRp at the same site, the active site. On the right-hand side of Figure 7, the box-plot graph represented the average binding energy $(\mathrm{Kcal} / \mathrm{mol})$ of all substances. Unfortunately, the result here was the opposite of the finding from the previous experiment, the proteinase. As could be seen in Figure 7 on the right-hand side, andrographolide showed the weakest binding energy whereas remdesivir exhibited 
http://wjst.wu.ac.th

the strongest binding as same as ATP, the native ligand. The last was favipiravir, which exhibited the middle binding capacity. The result here was also against the previous study, which claimed that the derivatives of andrographolide could strongly bind with the homology modeling protein structure of the SARS-CoV-2 RdRp [20]. However, one of the active derivatives that had been reported from the previous work was andrographoside, the glycoside form of andrographolide. The extra sugar moiety might play an important role here by increasing the binding affinity and mocking the native ligand since ATP also consists of sugar part in the molecule.

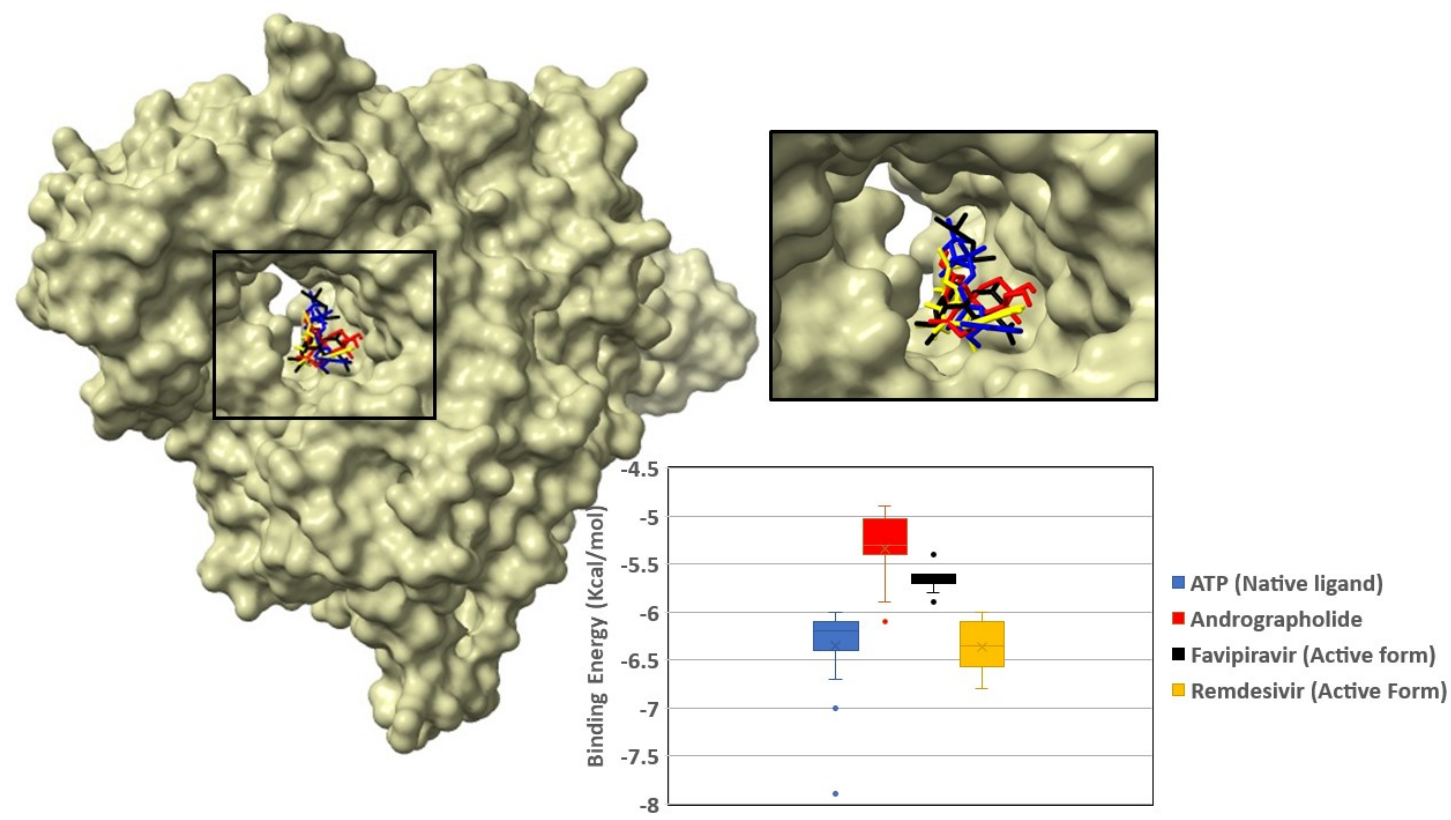

Figure 7 Molecular poses of ATP molecule (native ligand, blue color), andrographolide (red color), and the active forms of favipiravir (standard drug, black color) and remdesivir (standard drug, yellow color), and SARS-CoV-1 RdRp (PDB ID: 6NUR) as shown on the left-hand side. The comparison of the average binding energy $(\mathrm{Kcal} / \mathrm{mol})$ of ATP molecule, andrographolide, and the active forms of favipiravir and remdesivir as presented on the left-hand side.

To have a better understanding in the role of sugar in the molecular interation, the two dimentional diagram of the ligand-protein complex was performed and presented as could be seen in Figure 8. The interaction diagram had revealed that multiple amino acids had been involved in the molecualr interation. Even though Val557 as an active residue did not take part in these interactions but Asp623, Thr680, and Asn691, which were suggested to stabilize the ribose sugar of the new incoming nucleotide triphosphate or NTP by forming the hydrogen bondings at 2' and 3' -hydroxyl position were presented. This interaction plays a vital role in the replication of the new viral RNA strand [23]. The molecular diagram (Figure 8A) had also revealed that 2' and 3' -hydroxyl groups of the ribose moiety of ATP, the native ligand, could form the hydrogen bondings with Asp623. Moreover, ATP could also hydrophobically interacted with Ser682, Thr687, and Ala688, which were the neighboring residues of Thr680 and Asn691, respectively. Therefore, the molecular docking result here is in good agreement with the previous report [20] and shows the impact of the sugar moiety in the molecular interaction. Furthermore, the interaction diagram had also pointed out that the sugar part of the active form of both favipiravir and remdesivir, the standard drugs, could interact with Asp623 as could be seen in Figures 8B and 8C. Since both drugs were a nucleotide analog, the similar molecular interaction to ATP here was expected. However, favipiravir 
http://wjst.wu.ac.th

was the only one that could interact with Thr680 directly (Figure 8B). On the other hand, andrographolide could only form a hydrophobic interaction with Asp623. Even though, andrographolide could also interact with Asn691 but it did not have an impact on the bind affinity between andrographolide and RdRp. As discussed earlier in this section, the reason that andrographolide here could only form a weak interaction with Asp623, the key residue, might be because the sugar moiety was missing, Figure 8D. In conclusion, the interaction diagram had revealed that all compounds could bind at the active site of the RdRp enzyme but the type of the interactions was different, and the sugar moiety played an essential role in the molecular interaction between the ligand and Asp623.

A

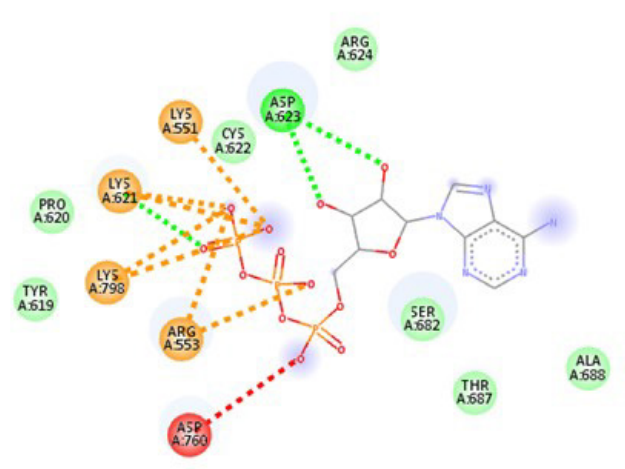

B

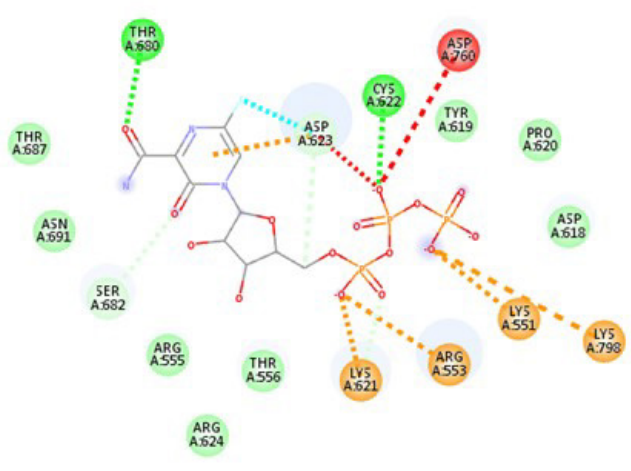

C

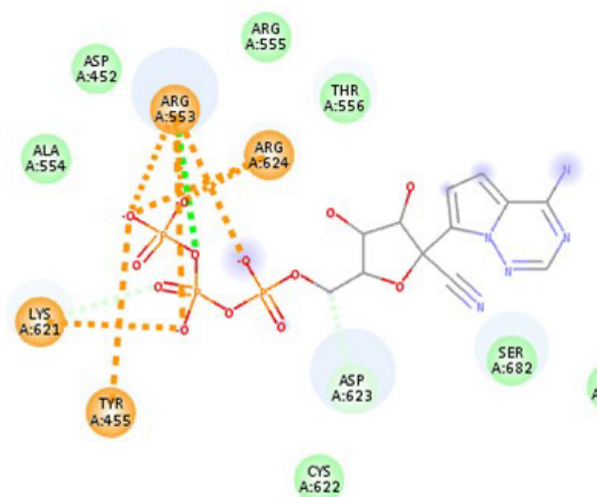

D

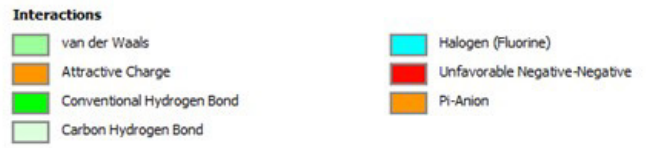

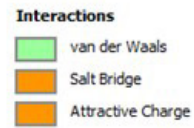

A:622
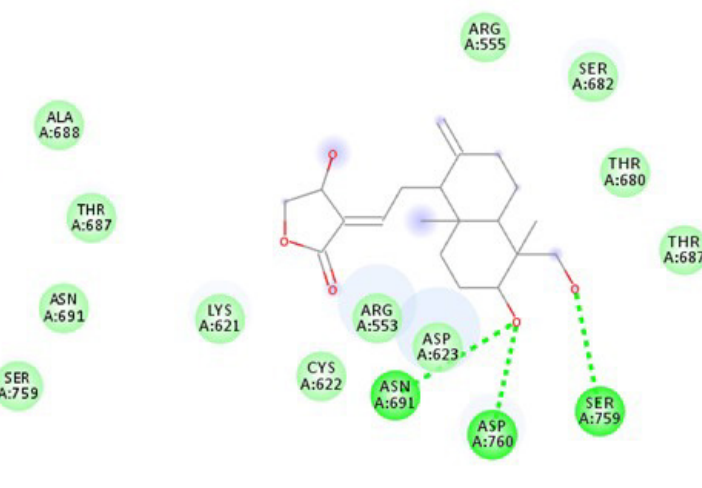
http://wjst.wu.ac.th

Figure 8 Interaction diagram between SARS-CoV-1 RdRp and ATP molecule as a native ligand (A), the active forms of favipiravir (B) and remdesivir as the standard drugs (C) and andrographolide (D).

\section{Conclusions}

Considerably, this study is one of the early studies that shed the light on the anti-SARS-CoV-2 activity of andrographolide, the well-known active metabolite from a common antiviral Thai herbal medicine like $A$. paniculata. This study has suggested that the antiviral proteinase is the possible mechanism of action of andrographolide, which can be used against the SARS-CoV-2 infection since andrographolide demonstrates the same potency as lopinavir, the standard antiviral proteinase drug. There may be an argument on the reliability of the computational techniques that had been applied in this study. It was not deniable that these was just a model. However, these techniques can provide insightful information if they are executed properly, for example, the application with commonly known antiviral regimens. This approach has been known as the computational drug repurposing method and has been used currently to identify the candidates for the SARS-CoV-2 treatment globally. Even if this is the case, further investigations are essential to confirm this activity since this is just an early step of drug research.

Interestingly, as mentioned also in the result section, combining the findings here from this study together with the previous reports suggested that receiving andrographolide from the natural source may not just only have a better effect since $A$. paniculata biologically synthesize a group of the andrographolide, the derivatives of andrographolide, such as andandrographoside (andrographolide glycoside), which was reported as a potential inhibitor against SARS-CoV-2 RdRp, but also provide a cheaper, quicker, easier to access, and, of course, safer antiviral regimen since A. paniculata has been used in the Thai primary health care system for a long time. Last but not least, until now, no report has been claimed the preventing effect of the andrographolide or its derivatives from the SARS-CoV-2 infection.

\section{Acknowledgments}

The author of this study would like to express his sincerely thanks to his family who has always been supportive. Also, special thanks to his friends who have encouraged him to perform and write this research.

\section{References}

[1] FA Rabi, MSA Zoubi, GA Kasasbeh, DM Salameh and ADA Nasser. SARS-CoV-2 and coronavirus disease 2019: What we know so far. Pathogens 2020; 9, 231.

[2] RA Neher, RA Dyrdak,V Druelle, EB Hodcroft and J Albert. Potential impact of seasonal forcing on a SARS-CoV-2 pandemic. Swiss. Med. Wkly. 2020; 150, 1-8.

[3] Y Zhou, Y Hou, J Shen, Y Huang, W Martin and F Cheng. Network-based drug repurposing for novel coronavirus 2019-nCoV/SARS-CoV-2. Cell. Discov. 2020; 6, 14.

[4] S Gupta, KP Mishra and L Ganju. Broad-spectrum antiviral properties of andrographolide. Arch. Virol. 2017; 162, 611-23.

[5] M Macchiagodena, M Pagliai and P Procacci. Inhibition of the main protease 3CL-pro of the Coronavirus Disease 19 via structure-based ligand design and molecular modeling. arXiv. preprint. 2020. https://doi.org/arXiv:2002.09937.

[6] AC Walls, YJ Park, MA Tortorici, A Wall, AT McGuire and D Veesler. Structure, function, and antigenicity of the SARS-CoV-2 spike glycoprotein. Cell 2020; 181, 281-92.

[7] MA Phillips, MA Stewart, DL Woodling and ZR Xie. Has molecular docking ever brought us a medicine. Molecular Docking. IntechOpen, London, UK, 2018, p. 141-78.

[8] X Zhu, Q Liu, L Du, L Lu and S Jiang. Receptor-binding domain as a target for developing SARS vaccines. J. Thorac. Dis. 2013; 5, 142-8.

[9] Notepad++, Available at: https://notepad-plus-plus.org, accessed March 2020. 
http://wjst.wu.ac.th

[10] M Gouy, S Guindon and O Gascuel. SeaView version 4: A multiplatform graphical user interface for sequence alignment and phylogenetic tree building. Mol. Biol. Evol. 2010; 27, 221-4.

[11] S Chetty and ME Soliman. Possible allosteric binding site on Gyrase B, a key target for novel antiTB drugs: Homology modelling and binding site identification using molecular dynamics simulation and binding free energy calculations. Med. Chem. Res. 2015; 24, 2055-74.

[12] EF Pettersen, TD Goddard, CC Huang, GS Couch, DM Greenblatt, EC Meng and TE Ferrin. UCSF Chimera--a visualization system for exploratory research and analysis. J. Comput. Chem. 2004; 25, 1605-12.

[13] O Trott and AJ Olson. AutoDock Vina: Improving the speed and accuracy of docking with a new scoring function, efficient optimization and multithreading. J. Comput. Chem. 2010; 31, 455-61.

[14] AD Hunter. ACD/ChemSketch 1.0 (freeware); ACD/ChemSketch 2.0 and its tautomers, dictionary, and 3D plug-ins; ACD/HNMR 2.0; ACD/CNMR 2.0. J. Chem. Educ. 1997; 74, 905-6

[15] MD Hanwell, DE Curtis, DC Lonie, T Vandermeersch, E Zurek and GR Hutchison. Avogadro: An advanced semantic chemical editor, visualization, and analysis platform. J. Cheminformatics. 2012; 4, 1-17.

[16] JH Jiang and P Deng. Discovery of new inhibitors of transforming growth factor-beta type 1 receptor by utilizing docking and structure-activity relationship analysis. Int. J. Mol. Sci. 2019; 20, 4090.

[17] L Zhang and R Zhou. Binding mechanism of remdesivir to SARS-CoV-2 RNA dependent RNA polymerase. Preprints 2020. https://doi.org/10.20944/preprints202003.0267.v1.

[18] J Lung, YS Lin, YH Yang, YL Chou, LH Shu, YC Cheng, HT Liu and CY Wu. The potential chemical structure of anti

J.SAR8- Cóbl: 2 RNA - dependent 2020; 92 693-7.

[19] Z Jin, X Du, Y Xu, Y Deng, M Liu, Y Zhao, B Zhang, X Li, L Zhang, C Peng and Y Duan. Structure of M pro from SARS-CoV-2 and discovery of its inhibitors. Nature 2020; 582, 289-93.

[20] C Wu, Y Liu, Y Yang, P Zhang, W Zhong, Y Wan, Q Wang, Y Xu, M Li, X Li and M Zheng. Analysis of therapeutic targets for SARS-CoV-2 and discovery of potential drugs by computational methods. Acta Pharm. Sin. B 2020; 10, 766-88.

[21] V Chandramohan, A Kaphle, M Chekuri, S Gangarudraiah and GB Siddaiah. Evaluating andrographolide as a potent inhibitor of NS3-4A protease and its drug-resistant mutants using in silico approaches. Adv. Virol. 2015; 4, 1-9.

[22] K Bafna, RM Krug and GT Montelione. Structural similarity of SARS-CoV-2 Mpro and HCV NS3/4A proteases suggests new approaches for identifying existing drugs useful as COVID-19 therapeutics. ChemRxiv 2020. https://dx.doi.org/10.26434\%2Fchemrxiv.12153615.

[23] RN Kirchdoerfer and AB Ward. Structure of the SARS-CoV nsp12 polymerase bound to nsp7 and nsp8 co-factors. Nat. Commun. 2019; 10, 2342. 


\title{
Supplementary file
}

The Antiviral Activity of Andrographolide, the Active Metabolite from Andrographis paniculata (Burm. f.) Wall. ex Nees. against the SARSCoV-2 by Using Bio- and Chemoinformatic Tools

\begin{abstract}
>Extracted SARS-CoV-2 RdRp sequence from the ORF 1ab polyproteins (GenBank: QHD43415.1) SADAQSFLNRVCGVSAARLTPCGTGTSTDVVYRAFDIYNDKVAGFAKFLKTNCCRFQEKD EDDNLIDSYFVVKRHTFSNYQHEETIYNLLKDCPAVAKHDFFKFRIDGDMVPHISRQRLT KYTMADLVYALRHFDEGNCDTLKEILVTYNCCDDDYFNKKDWYDFVENPDILRVYANLGE RVRQALLKTVQFCDAMRNAGIVGVLTLDNQDLNGNWYDFGDFIQTTPGSGVPVVDSYYSL LMPILTLTRALTAESHVDTDLTKPYIKWDLLKYDFTEERLKLFDRYFKYWDQTYHPNCVN CLDDRCILHCANFNVLFSTVFPPTSFGPLVRKIFVDGVPFVVSTGYHFRELGVVHNQDVN LHSSRLSFKELLVYAADPAMHAASGNLLLDKRTTCFSVAALTNNVAFQTVKPGNFNKDFY DFAVSKGFFKEGSSVELKHFFFAQDGNAAISDYDYYRYNLPTMCDIRQLLFVVEVVDKYF DCYDGGCINANQVIVNNLDKSAGFPFNKWGKARLYYDSMSYEDQDALFAYTKRNVIPTIT QMNLKYAISAKNRARTVAGVSICSTMTNRQFHQKLLKSIAATRGATVVIGTSKFYGGWHN MLKTVYSDVENPHLMGWDYPKCDRAMPNMLRIMASLVLARKHTTCCSLSHRFYRLANECA QVLSEMVMCGGSLYVKPGGTSSGDATTAYANSVFNICQAVTANVNALLSTDGNKIADKYV RNLQHRLYECLYRNRDVDTDFVNEFYAYLRKHFSMMILSDDAVVCFNSTYASQGLVASIK NFKSVLYYQNNVFMSEAKCWTETDLTKGPHEFCSQHTMLVKQGDDYVYLPYPDPSRILGA GCFVDDIVKTDGTLMIERFVSLAIDAYPLTKHPNQEYADVFHLYLQYIRKLHDELTGHML DMYSVMLTNDNTSRYWEPEFYEAMYTPHTV
\end{abstract}

Figure S1 Extracted amino acid sequences of the SARS-CoV-2 RdRP from the ORF 1ab polyproteins. 
http://wjst.wu.ac.th

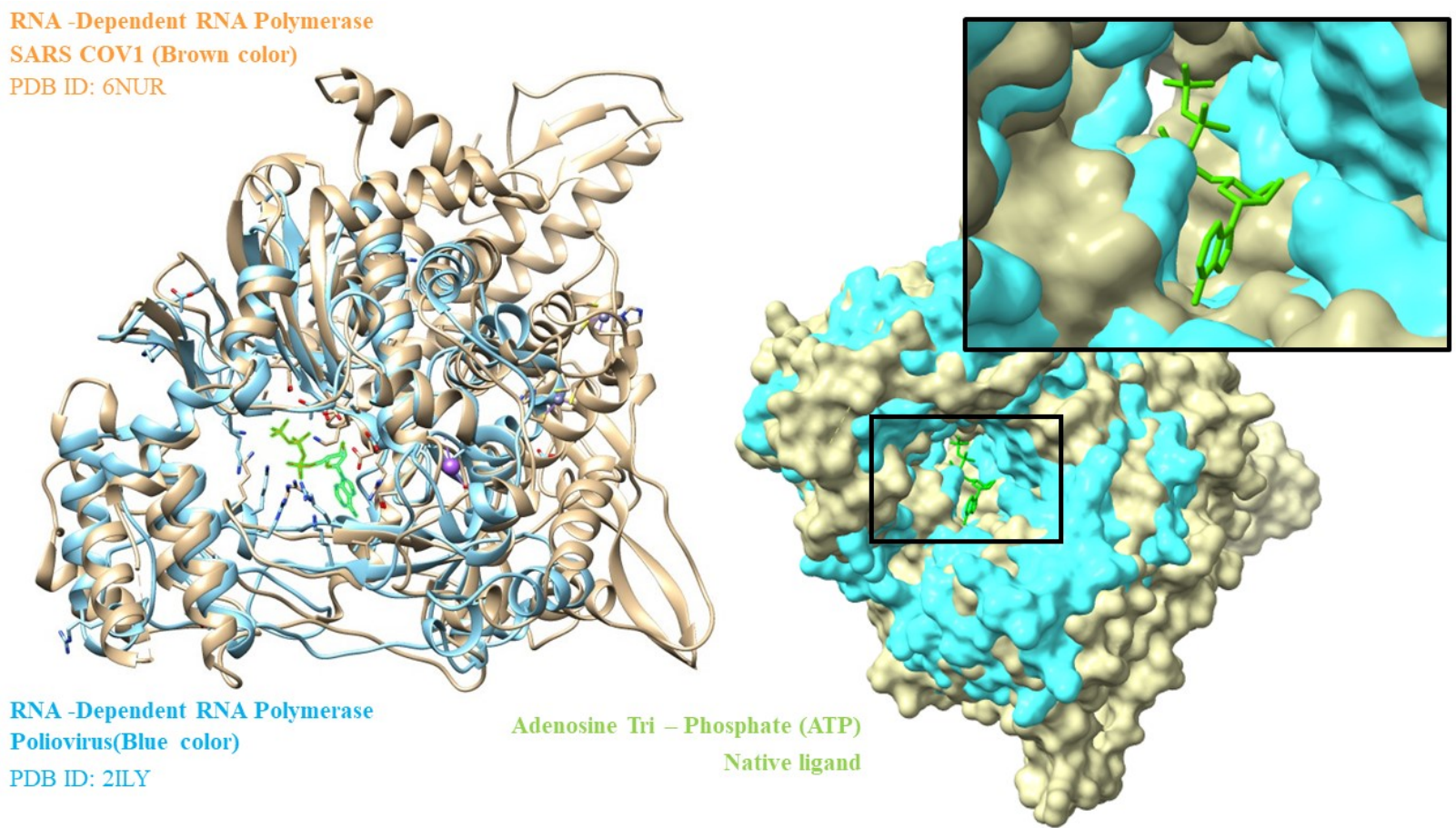

Figure S2 3D structures alignment between SARS-CoV-1 RdRp (PDB ID: 6NUR) and poliovirus RdRp (PDB ID: 2ILY). ATP as a native ligand (green color) from poliovirus RdRp was used to navigate the binding pocket of SARS-CoV-1 RdRp. On the left-hand side exhibited the protein alignment in a ribbon format while on the right-hand side exhibited the protein alignment in a surface format. The upper right corner presented the close-up photo in the binding pocket. 


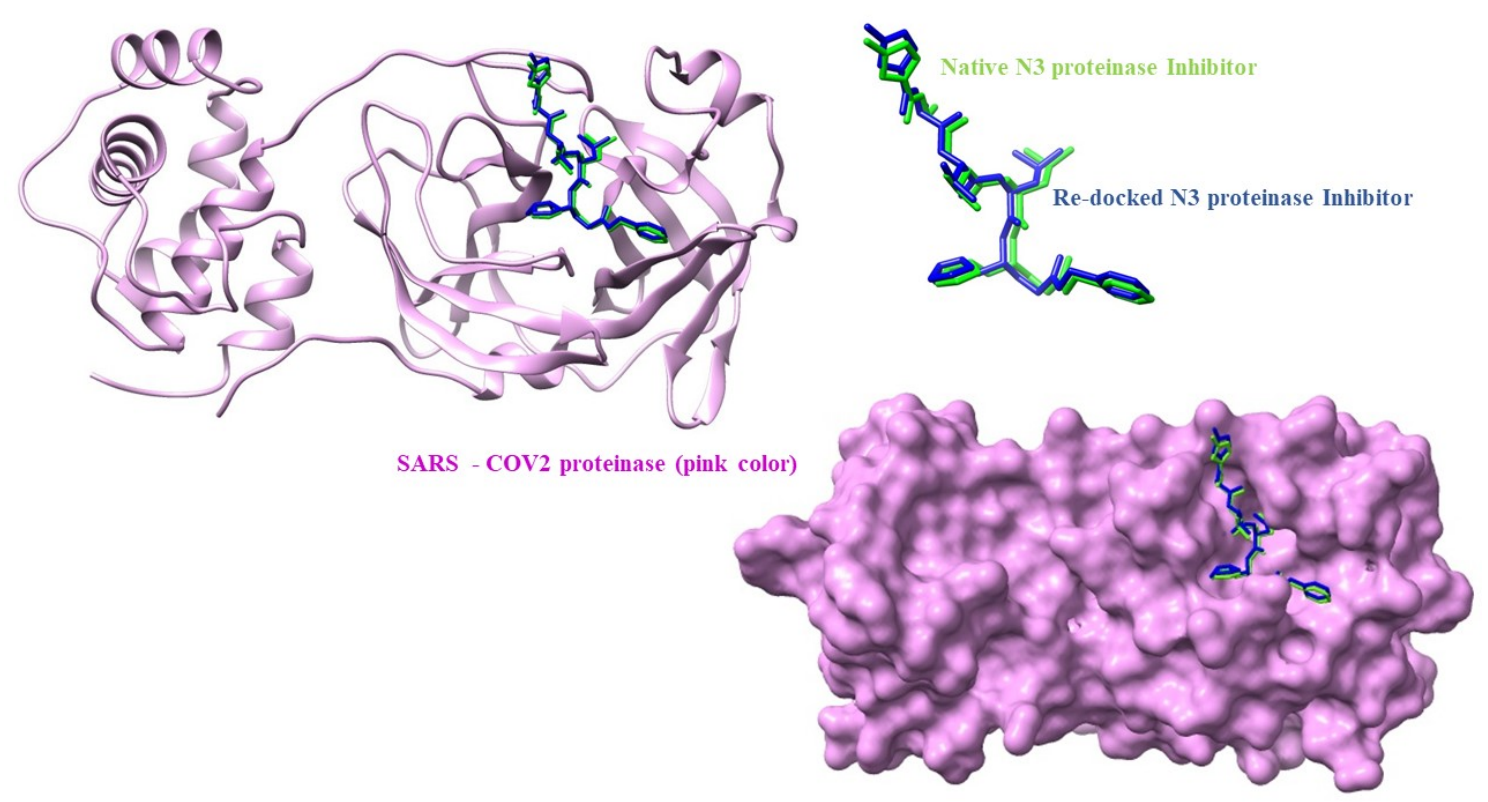

Figure S3 The validation of the molecular docking method from the SARS-CoV-2 proteinase experiment. Native N3 proteinase inhibitor, before extraction as in green color, and re-docked N3 proteinase inhibitor, after redocking as in blue color. The alignment of the native and re-docked N3 indicated that this method passed the acceptance criteria.
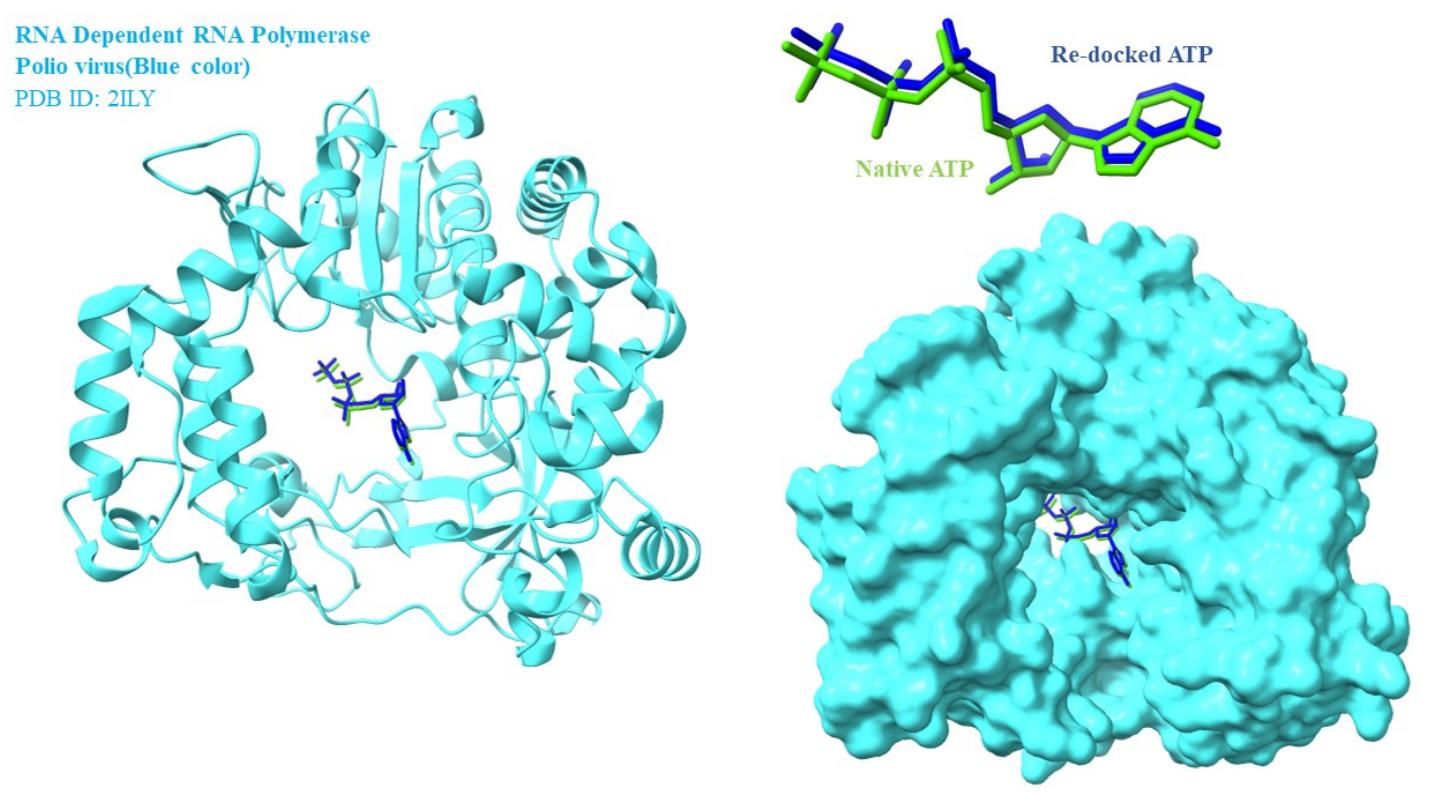

Figure S4 The validation of the molecular docking method from the polioviral RdRp experiment. Native ATP, before extraction as in green color and re-docked ATP, after redocking as in blue color. The alignment of native and re-docked ATP indicated that this method also passed the acceptance criteria. 